\title{
Entropic Origin of the Attenuated Width of Ice-Water Interface
}

\author{
Saumyak Mukherjee and Biman Bagchi* \\ Solid State and Structural Chemistry Unit \\ Indian Institute of Science, Bengaluru - 560012, India \\ *Email: bbagchi@iisc.ac.in
}

S1. Correlation between tetrahedral order parameter and number of hydrogen bond defects

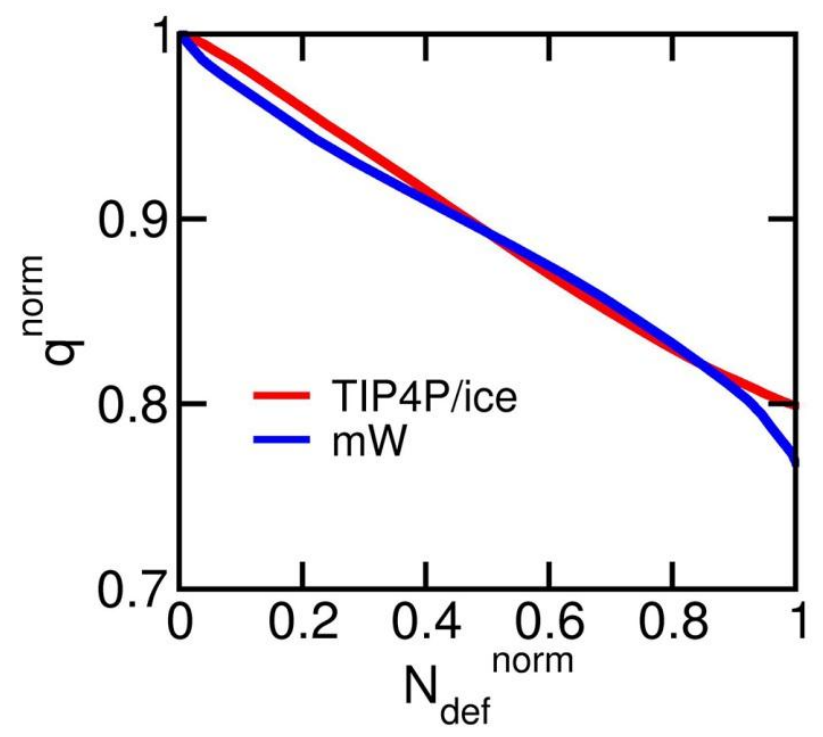

Figure S1. Correlation between tetrahedral order parameter and number of hydrogen bond defects across the solid-liquid interface. These two parameters are strongly anti-correlated with an correlation coefficient of -0.99 in both TIP4P/ice and $\mathrm{mW}$ water models. 


\section{S2. Correlation between structure, dynamics and thermodynamics}
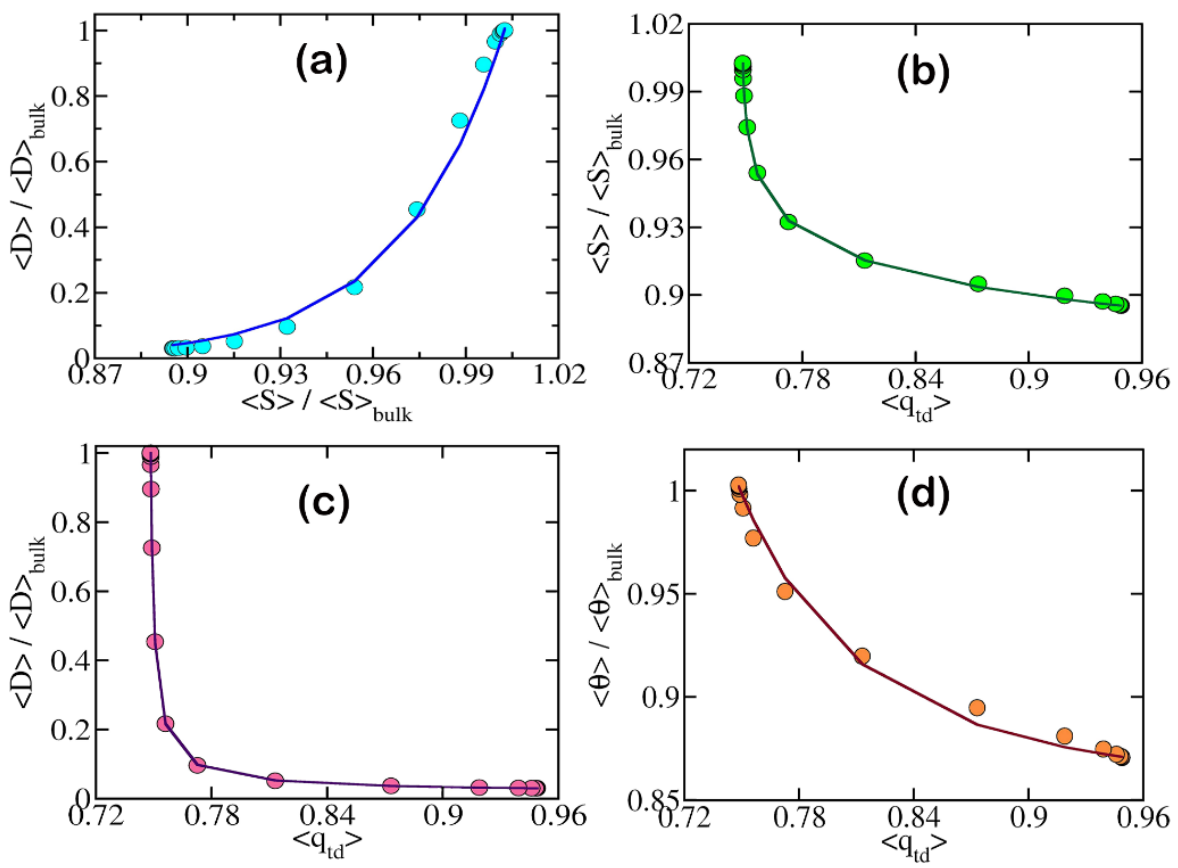

Figure S2. Relationship between different order parameters in the ice-water coexistence system. (a) Diffusion coefficient against entropy, (b) entropy against tetrahedrality order parameter, (c) diffusion against tetrahedrality order parameter, and (d) angular jump against tetrahedrality order parameter.

The order parameters described so far show correlated / anticorrelated changes across the ice-water interface. Here we explore the mutual relationships between these parameters. Figure S2 shows these correlated changes for (a) diffusion coefficient and entropy, (b) entropy and tetrahedral order parameter, (c) diffusion coefficient and tetrahedral order parameter, and (d) angular displacement and tetrahedral order parameter.

Surprisingly, diffusion and entropy show an exponential relationship, akin to the celebrated Rosenfeld scaling. The plot in Figure S3a is fitted to the following equation.

$$
\frac{\langle D\rangle}{\langle D\rangle_{\text {bulk }}}=a e^{b\left(\langle S\rangle /\langle S\rangle_{b u l k}\right)}
$$

Here, $b=29.9$. 
The graphs in Figure S3b, Figure S3c and Figure S3d are fitted to the following power law function.

$$
y=a_{1}\left(\frac{\left\langle q_{t d}\right\rangle-a_{2}}{a_{3}}\right)^{-a_{4}}+a_{5}
$$

The fitting parameters for the relationship of entropy, diffusion coefficient, and angular jump against tetrahedral order parameter are given in Table S1.

Table S1. Fitting parameters for the power law relationship of entropy, diffusion and angular jump with tetrahedral order parameter.

\begin{tabular}{|c|c|c|c|c|c|}
\hline $\mathbf{y}$ & $\boldsymbol{a}_{\mathbf{1}}$ & $\boldsymbol{a}_{\mathbf{2}}$ & $\boldsymbol{a}_{\mathbf{3}}$ & $\boldsymbol{a}_{\mathbf{4}}$ & $\boldsymbol{a}_{\mathbf{5}}$ \\
\hline$\langle\boldsymbol{S}\rangle /\langle\boldsymbol{S}\rangle_{\text {bulk }}$ & 0.66 & 0.75 & 0.64 & 0.03 & 0.22 \\
\hline$\langle\boldsymbol{D}\rangle /\langle\boldsymbol{D}\rangle_{\text {bulk }}$ & 0.04 & 0.75 & 0.06 & 0.86 & 0.02 \\
\hline$\langle\boldsymbol{\theta}\rangle /\langle\boldsymbol{\theta}\rangle_{\text {bulk }}$ & 0.08 & 0.54 & 0.25 & 3.30 & 0.85 \\
\hline
\end{tabular}




\section{S3. Derivative plot of order parameters in ice-water system}
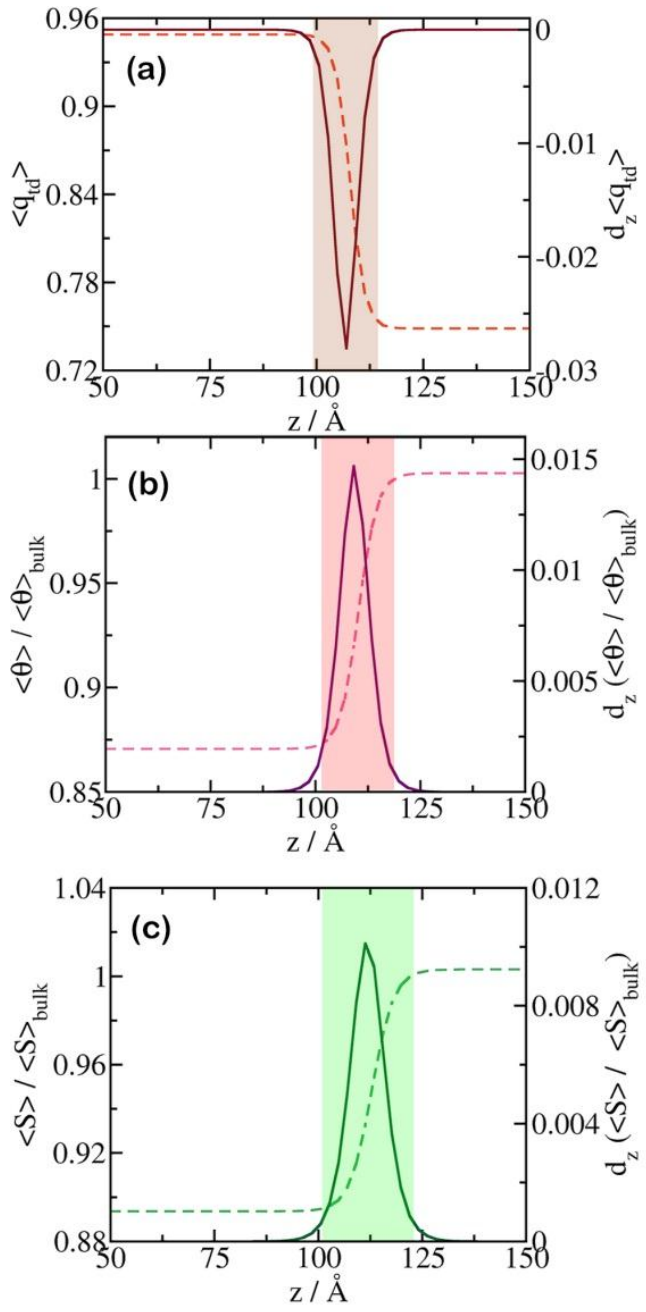

Figure S3. Position and width of ice-water interface represented by the derivative of the concerned variable. The change of the variable along the z-axis is shown by the broken lines. The order parameters are (a) tetrahedrality order parameter, (b) angular jump in $100 \mathrm{fs}$, and (c) entropy. The peak position and the width of the derivative peak give the position and the width of the ice-water interface respectively. Clearly, the interface width depends on the order parameter used to define it. 
S4. Comparison of interfacial widths with respect to the three faces of ice-1h

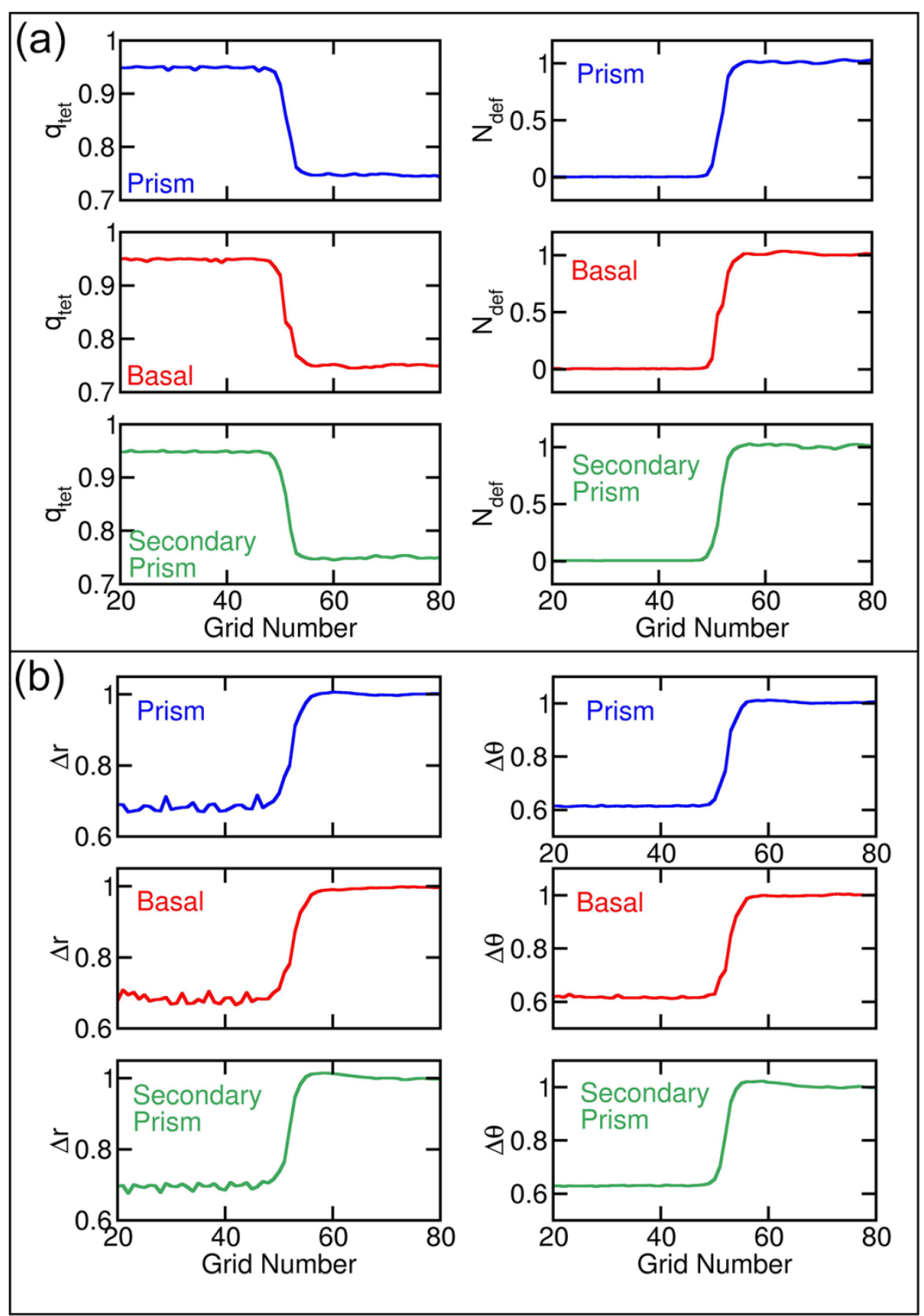

Figure S4. (a) Structural (tetrahedral order parameter and number of hydrogen bond defects) and (b) dynamical (translational and rotational displacement in $1 \mathrm{ps}$ ) plotted as functions of the three coordinate axes, perpendicular to the solid-liquid interface. In each case, the interface is any of 
the three faces of hexagonal ice (prismatic, basal and secondary prismatic). The interfacial width varies negligibly with respect to the ice crystal face. 